\title{
Modélisation de poches de cavitation par une méthode de suivi d'interfaces de type VOF
}

\author{
L. Dieval ( $\left.{ }^{1}\right)$, R. Marcer $\left({ }^{2}\right)$, M. Arnaud ( $\left.{ }^{1}\right)$
}

(1) Centre Technique des Systèmes Navals, Toulon

(2) Principia Recherche et Développement, La Seyne/Mer

\section{IINTRODUCTION}

Les écoulements cavitants ont fait l'objet de nombreuses modélisations numériques, notamment l'étude de la cavitation par poche a été appréhendée par différents types de méthode : les méthodes potentielles, les méthodes de suivi d'interface et les méthodes diphasiques. Ces méthodes ont des degrés de complexité divers et des domaines de validité bien spécifiques.

Pour l'étude de la cavitation stationnaire, on utilise généralement des méthodes de singularités en écoulement potentiel car elles permettent de décrire précisément l'interface d'une poche si sa géométrie est simple, à partir d'un processus itératif qui permet d'obtenir à convergence une ligne de courant moyenne à pression constante. Le sillage de la cavité qui est une zone instationnaire et diphasique n'est cependant pas reproduit par ce type de modèle, et une loi de sillage empirique est alors nécessaire pour recoller la ligne de courant sur le profil.

Pour la cavitation instationnaire, les méthodes les plus performantes sont actuellement les méthodes de type diphasique. Ces modèles calculent généralement l'écoulement d'un fluide moyen compressible où la densité varie fortement à l'interface de la cavité [1], [2], [3]. Certains auteurs utilisent une loi d'état exacte ainsi qu'une équation d'énergie [2]. D'autres traitent le fluide comme un fluide moyen compressible fictif et introduisent un taux de vide pour distinguer les phases liquide et vapeur incompressibles, la zone de transition étant compressible et obéissant à une loi d'état fictive reliant la densité à la pression [3].

L'objectif de l'étude est de démontrer les capacités de la méthode VOF à simuler les phénomènes de cavitation instationnaire, notamment les processus de création, de régénération, de suivi et de fractionnement de poche. Cette méthode consiste à définir, dans un maillage fixe, l'interface par la fraction volumique des deux phases liquide et gazeuse [4]. Son avantage par rapport aux méthodes purement diphasiques est qu'elle constitue une vraie méthode de suivi d'interface sans diffusion numérique et ne présentant pas la raideur mathématique propre aux modèles diphasiques liée au traitement des fluides compressibles lorsque la vitesse du son est très supérieure à la vitesse de l'écoulement.

L'écoulement liquide est simulé par un code de mécanique des fluides développé à Principia [5], [6] qui résout les équations de Navier-Stokes ou d'Euler. L'évolution de l'interface liquide-gaz des poches est calculée par la méthode VOF à partir d'une équation de transport pour la fraction volumique.

\section{II — MODÉLISATION NUMÉRIQUE}

\subsection{Equations}

Les équations décrivant l'écoulement sont les équations d'Euler qui régissent les écoulements de fluide parfait. Elles s'écrivent pour un fluide incompressible, en écoulement bidimensionnel plan et en coordonnées cartésiennes :

$$
\frac{\partial W}{\partial t}+\frac{\partial G}{\partial x}+\frac{\partial H}{\partial y}=R
$$

avec

$$
W=\left[\begin{array}{l}
0 \\
\rho u \\
\rho v
\end{array}\right] ; G=\left[\begin{array}{l}
\rho u \\
\rho u^{2}+p \\
\rho u v
\end{array}\right] ; H=\left[\begin{array}{l}
\rho v \\
\rho v u \\
\rho v^{2}+p
\end{array}\right] ; R=\left[\begin{array}{l}
0 \\
0 \\
-\rho g
\end{array}\right]
$$

où $u$ et $v$ sont les composantes cartésiennes de la vitesse dans les directions $x$ et $y$, $\rho$ la masse volumique constante du liquide, $p$ la pression et $g$ l'accélération de la pesanteur.

$S i$ l'on considère un maillage curviligne fixe défini par un système de coordonnées curviligne $(\xi, \eta)$, l'équation (1) s'écrit à l'aide de ces nouvelles coordonnées, de la façon suivante :

$$
\frac{1}{J} \frac{\partial W}{\partial t}+\frac{\partial \bar{G}}{\partial \xi}+\frac{\partial \bar{H}}{\partial \eta}=\frac{1}{J} R
$$

où les flux transformés $\bar{G}$ et $\bar{H}$, les composantes contravariantes $\tilde{u}$ et $\tilde{v}$, et le jacobien de la transformation de coordonnées $J$ sont respectivement :

$$
\begin{aligned}
& \bar{G}=\frac{1}{J}\left[\begin{array}{l}
\rho \tilde{u} \\
\rho \tilde{u} u+\xi_{x} p \\
\rho \tilde{u} v+\xi_{y} p
\end{array}\right] \bar{H}=\frac{1}{J}\left[\begin{array}{l}
\rho \tilde{v} \\
\rho \tilde{v} u+\eta_{x} p \\
\rho \tilde{v} v+\eta_{y} p
\end{array}\right] \\
& \tilde{u}=\xi_{x} u+\xi_{y} v \\
& \tilde{v}=\eta_{x} u+\eta_{y} v \\
& J=\frac{\partial(\xi, \eta)}{\partial(x, y)}
\end{aligned}
$$




\subsection{Pseudo-compressibilité}

La discrétisation instationnaire est réalisée à l'aide d'un schéma en temps totalement implicite du second ordre. La résolution du système d'équations pour les inconnues au pas de temps $n+1$ repose sur une technique de pseudo-compressibilité [5].

Dans les équations discrétisées à l'instant $\mathrm{n}+1$, on introduit des dérivées par rapport à une variable $\tau$, dite pseudo-temps, qui font intervenir une nouvelle inconnue $\tilde{\rho}$ dite pseudo-masse volumique et assujettie à rester positive :

$$
\frac{1}{J} \frac{\partial \tilde{W}^{n+1}}{\partial \tau}+\frac{13 W^{n+1}+4 W^{n}+W^{n-1}}{2 \Delta t}+\left(\frac{\partial \bar{G}}{\partial \zeta}\right)^{n+1}+\left(\frac{\partial \bar{H}}{\partial \eta}\right)^{n+1}=\frac{1}{J} R
$$

avec :

$$
\tilde{W}^{n+1}=\tilde{\rho}\left[\begin{array}{l}
1 \\
u \\
v
\end{array}\right]^{n+1}=\frac{\tilde{\rho}}{\rho} W^{n+1}
$$

L'introduction de la variable $\tilde{\rho}$ nécessite une équation supplémentaire qui s'identifie à une pseudo-loi d'état permettant de calculer la pression en fonction de $\tilde{\rho}$. Cette relation peut être choisie de diverses façons. Comme discuté dans [5], le choix retenu est le suivant

$$
p=\rho U_{0}^{2} \ln \frac{\tilde{\rho}}{\rho_{\text {ref }}}+P_{\text {ref }}
$$

où $U_{0}, P_{r e f}$ et $\rho_{\text {ref }}$ sont des constantes.

Le système est hyperbolique par rapport à $\tau$ (c'est l'une des raisons qui ont conduit à choisir ce système) et il est formellement très voisin des équations d'Euler en fluide compressible (sans l'équation de l'énergie), grâce à la présence du même facteur $\tilde{\rho}$ dans les termes de dérivées en $\tau$. Cette propriété permet d'utiliser, pour sa résolution, des algorithmes existants et efficaces qui ont été développés pour les écoulements compressibles. On a mis en œuvre, pour la résolution du système d'équations précédent, une adaptation de la méthode de volumes finis, avec viscosité artificielle, développée à l'origine par Jameson $e t$ al. [7] pour les écoulements de fluides compressibles.

La discrétisation en espace est de type centré sur un maillage structuré. Les variables $\tilde{\rho}, u$, $v$, et $p$ étant définies aux centres des cellules, les flux aux interfaces sont obtenus à partir des moyennes des variables aux centres des deux cellules adjacentes. La viscosité artificielle, nécessaire dans le cas d'un schéma centré pour assurer la stabilité, la convergence et éviter le découplage des nœuds pair et impair, comporte des dérivées du second ordre et des dérivées du quatrième ordre [7]. Elle est ajustée au moyen de coefficients.

La résolution en pseudo-temps est basée sur le schéma de Runge-Kutta à cinq étapes, avec utilisation d'un pseudo pas de temps local et un lissage implicite des résidus [7]. Le schéma de base de Runge-Kutta est explicite, mais on introduit un traitement implicite du terme source instationnaire $\partial W / \partial t$, ce qui renforce la stabilité tout en laissant la résolution effectivement explicite.

La valeur maximale du pas en pseudo-temps $\Delta \tau$ est fixée par un critère de stabilité local du type Courant-FriedrichsLewy. On utilise pour chaque maille la valeur maximale locale du pseudo pas de temps. La méthode est inconditionnellement stable en temps physique et est précise au second ordre. On trouve une présentation cette méthode numérique dans [8].

\section{Méthode VOF}

Les interfaces liquide-gaz sont des frontières libres dont les positions sont des inconnues du problème. Dans la méthode VOF, le problème est discrétisé dans un maillage fixe, et la surface libre n'est connue que par une variable scalaire $F$ définie dans chaque maille et qui est la fraction de volume de la maille occupée par le fluide. Cette variable $F$ prend donc des valeurs comprises entre 0 et 1 , bornes incluses, et l'interface est localisée dans les cellules où l'on a $0<F<1$.

L'évolution de la variable $F$ dans une maille $\Omega$ est gouvernée par l'équation de bilan de masse de cette maille que l'on peut écrire :

$$
V \frac{d F}{d t}+=\int_{\partial \Omega} f \vec{U} \cdot \vec{n} d S=0
$$

où $V$ est le volume de la maille, $\partial \Omega$ sa frontière et où $f$ une fonction définie en tout point et ne prenant que deux valeurs : 1 en un point occupé par le fluide, et 0 autrement.

Le problème que pose l'intégration de (5) provient du fait que $f$ n'est pas connue ou plus précisément n'est connue que par son intégrale $F V$ dans chaque cellule. L'originalité et l'intérêt de la méthode VOF résident dans l'évaluation approchée, mais conservative, du flux de masse au travers de la seule connaissance de $F$ dans la maille $\Omega$ et dans les mailles voisines. Pour cette évaluation, on restreint l'orientation de l'interface dans la maille $\Omega$ à ne prendre que deux valeurs (en 2D), à savoir parallèle à l'une ou l'autre des faces de la cellule. En maillage cartésien, le critère de sélection se fait en évaluant les pentes locales par rapport aux deux dimensions $x$ et $y$, par différences finies sur $F$, pour une interface localement plane.

Considérons deux cellules adjacentes, ayant donc une interface commune. La composante normale de la vitesse permet de distinguer la cellule donneur (amont) et la cellule accepteur (aval). Le flux de masse à travers l'interface est alors déterminé selon un algorithme assez complexe qui distingue un certain nombre de situations possibles en fonction de la valeur de $F$ et de l'orientation de l'interface dans la cellule donneur et dans la cellule accepteur [4].

L'algorithme de calcul de $F$ est explicite en temps. La stabilité de cet algorithme limite le pas de temps à une valeur qui correspond à un déplacement de la surface libre d'une maille au plus. En pratique, cela signifie qu'une cellule pleine ne peut pas devenir vide (ou inversement) sur un pas de temps.

La méthode originale [4] a été conçue pour un maillage cartésien. Nous l'avons étendue en maillage curviligne de la façon suivante. Dans un maillage curviligne $(\xi, \eta)$, la cellule $\Omega$ est définie par les faces :

$$
\begin{aligned}
& \xi=\xi_{0} \text { et } \xi=\xi_{0}+\Delta \xi \\
& \eta=\eta_{0} \text { et } \eta=\eta_{0}+\Delta \eta
\end{aligned}
$$

L'équation de bilan de masse (5) pour $\Omega$ devient donc pour un maillage curviligne :

$V \frac{d F}{d t}+\left[\int_{\partial \Omega_{\xi_{+}}} \frac{f \tilde{u}}{J} d \eta-\int_{\partial \Omega_{\xi_{-}}} \frac{f \tilde{u}}{J} d \eta+\int_{\partial \Omega_{\eta_{+}}} \frac{f \tilde{v}}{J} d \xi-\int_{\partial \Omega_{\eta_{-}}} \frac{f \tilde{v}}{J} d \xi\right]=0$

On aboutit formellement à la même expression qu'en maillage cartésien, c'est-à-dire que l'on peut considérer $\xi$, $\eta$ comme des coordonnées cartésiennes à condition de remplacer les composantes cartésiennes de la vitesse par les composantes contravariantes modifiées par la division par $J$. 


\subsection{Traitement numérique sur l'interface}

Les conditions limites sur l'interface concernent les cellules partielles c'est-à-dire les cellules dont la valeur de $F$ est comprise entre 0 et 1 . Pour ces cellules, on extrapole les deux composantes de la vitesse à partir des valeurs de la vitesse sur les huit cellules voisines. Cette extrapolation repose sur une moyenne pondérée par une puissance de $F$ des vitesses dans les huit cellules voisines. La pression imposée dans ces mailles frontières correspond à la pression de vapeur régnant dans le domaine gazeux.

\subsection{Application du critère de cavitation}

Le calcul de la création et de l'évolution d'une poche de cavitation attachée au profil est simulé en deux étapes. La première consiste à effectuer un calcul stationnaire non cavitant, donc sans faire intervenir la méthode VOF. Le calcul a pour but de mettre en évidence le point de pression minimale, Pmin, sur le profil.

Le second calcul d'évolution de poche est alors initialisé par le champ dynamique issu du premier calcul et par une géométrie de poche de très petite dimension (fixée arbitrairement à deux mailles) imposée dans la région de pression minimale. Dans cette poche, les cellules du maillage sont considérées comme étant gazeuses, donc de VOF $F=0$, et à la pression de vapeur saturante. La valeur de Pmin impose le nombre de cavitation initial du calcul qui correspond donc à la cavitation naissante. On fait décroître ensuite au cours du calcul la pression en amont du canal, afin d'obtenir le nombre de cavitation souhaité.

La vitesse de déplacement de l'interface est égale à la vitesse normale de la phase liquide à laquelle s'ajoute une vitesse due au transfert de masse entre les phases. Cette dernière peut être habituellement négligée et on calcule l'évolution de l'interface par la méthode VOF qui fait intervenir le champ de vitesse local. En outre, à chaque pas de temps du calcul, un test sur le critère de cavitation permet de localiser d'éventuelles nouvelles cellules liquides de pression inférieure à la pression de vapeur, qui sont alors imposées comme étant gazeuses. Ce test qui consiste finalement à prendre en compte de manière semi-empirique le transfert de masse à l'interface liquide-gaz, gère ainsi de manière automatique une possible réalimentation de la poche gazeuse en fonction des conditions dynamiques locales de l'écoulement.

\section{III — RÉSULTATS}

Les calculs de cavitation présentés ont été réalisés sur deux types de profil. Un profil à bord d'attaque pointu présentan un extrados plat et un intrados bombé (profil 1) et un profil NACA (profil 2). Les calculs sont réalisés en utilisant les fonctions multidomaines du code. Les maillages de type $H$, similaires pour les deux profils, sont composés de deux sous-domaines, le premier $(141 \times 41$ cellules $)$ pour la discrétisation de la zone au dessus de l'extrados, le second $(141 \times$ 21 cellules) pour la partie en dessous de l'intrados. Les frontières entrante et sortante du domaine sont imposées à une distance équivalente à trois cordes, en amont et en aval du profil. Le point de pression minimale, issu d'un premier calcul non cavitant, fixe la cavitation naissante. Celle-ci se trouve dans tous les cas à l'extrados immédiatement en aval du bord d'attaque du profil.

\subsection{Profil 1}

Une expérimentation en canal hydrodynamique a permis de schématiser les différentes formes de cavitation en fonction de l'incidence du profil et du nombre de cavitation [9]. On s'intéresse ici à un cas de cavitation partielle où la longueur de la poche ne couvre qu'une partie du profil. Les conditions pour le calcul sont alors les suivantes:

Nombre de cavitation $\quad \sigma_{y}=0,7$

Incidence du profil $\quad \alpha=1$ degré

Vitesse de l'écoulement $\quad U_{\infty}=5 \mathrm{~m} / \mathrm{s}$

Corde du profil $\quad c=200 \mathrm{~mm}$

Pas de temps de la simulation $\Delta t=10^{-4} \mathrm{~s}$

La figure 1 montre la forme de la poche pour 2 instants : $138 \Delta t$ et $394 \Delta t$. On note globalement les caractéristiques spécifiques suivantes (confirmée par l'expérience) : une longueur faible de la cavité de l'ordre de $1 / 8$ de la corde du profil, la création cyclique de jets rentrants qui viennent fractionner la poche principale, le lâché d'une poche tourbillonnaire tournant en sens inverse trigonométrique, une implosion rapide de la poche fractionnée et finalement une certaine stabilité de longueur de la cavité principale.

\subsection{Profil 2}

Deux calculs de cavitation sont présentés pour ce profil. Les conditions de l'écoulement sont les suivantes :

Calcul 1:

Nombre de cavitation

Incidence du profil

Vitesse de l'écoulement

Corde du profil

Pas de temps de la simulation $\Delta t=300 \mathrm{~mm}$

Calcul 2:

$$
\begin{array}{ll}
\sigma=0,2 & \sigma=0,76 \\
\alpha=3,5 \text { degrés } & \alpha=3,5 \text { degrés } \\
U_{\infty}=6 \mathrm{~m} / \mathrm{s} & U_{\infty}=6 \mathrm{~m} / \mathrm{s} \\
c=300 \mathrm{~mm} & c=300 \mathrm{~mm}
\end{array}
$$

La figure 2 (conditions du calcul 1) montre d'une part la naissance et d'autre part l'aspect pleinement développé de la poche dans un cas de supercavitation. Le nombre de cavitation naissante donné par le calcul d'initialisation subcavitant, est de 1,72. L'écoulement transitoire est piloté à partir d'une décroissance linéaire du nombre de cavitation au cours du calcul, sur 20 pas de temps. La poche recouvre finalement la totalité du profil et présente une forte épaisseur surtout dans la zone de fermeture de la poche sur le bord de fuite du profil.
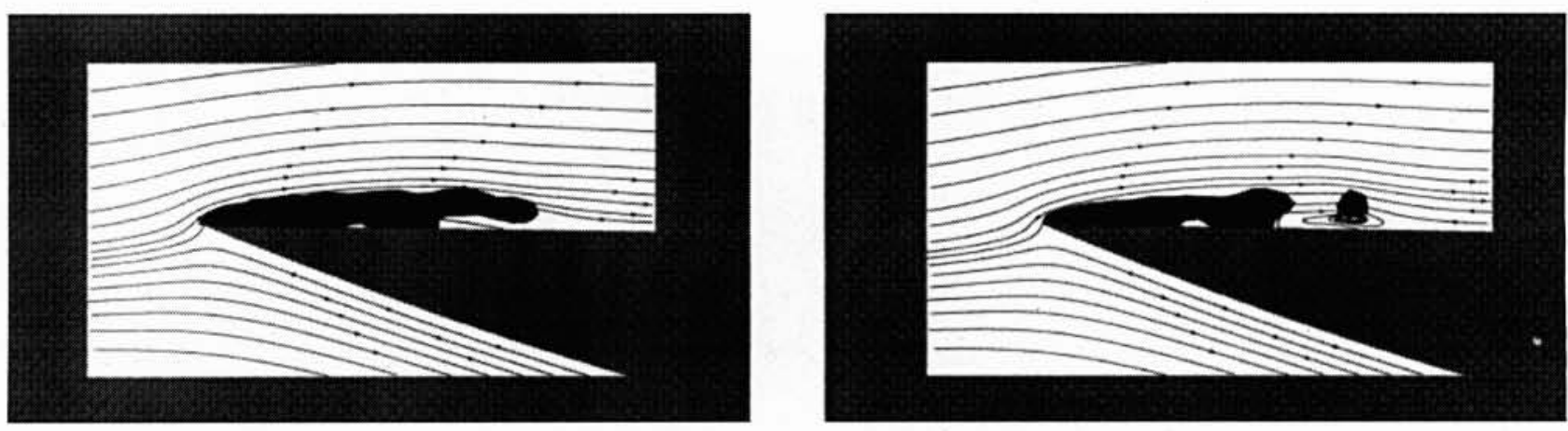

1. Cavitation partielle. 


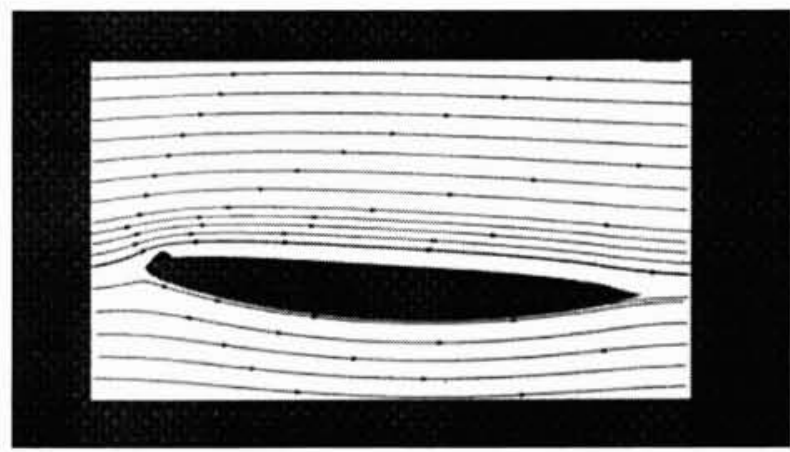

2. Evolution de la poche dans le cas de supercavitation.

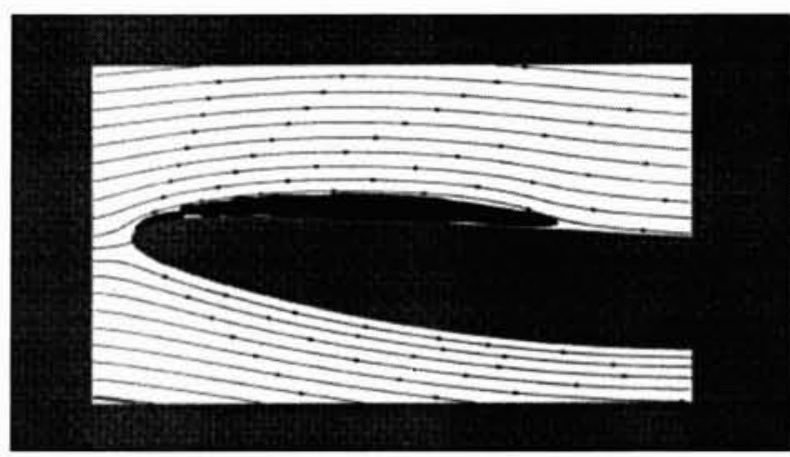

3. Jet rentrant.

La figure 3 montre la forme de la poche de cavitation pour les conditions d'écoulement du calcul 2. L'interface de la cavité suit une ligne de courant et atteint finalement une forme quasi-stationnaire. On note seulement quelques fluctuations dans la zone de recollement de la cavité induit par la présence d'un jet rentrant. Mais ces fluctuations ont toutefois une amplitude suffisamment faible pour ne pas provoquer de fractionnement de poches secondaires.

La validation du modèle par comparaisons avec des résultats expérimentaux fournis par la DRET sur ce profil, est en cours. Les premières comparaisons montrent un bon accord calcul-expérience.

\section{IV $\square$ CONCLUSIONS}

Les premiers calculs de poches de cavitation par une méthode VOF présentés dans cet article sont particulièrement encourageants. Les résultats démontrent que la méthode VOF et la méthodologie de calcul sont bien adaptées pour traiter ce phénomène. Ils mettent en évidence plus particulièrement les capacités de la méthode à simuler le gonflement de la poche, la formation de jets rentrants, les processus de fractionnement totalement instationnaires dans la zone aval de fermeture de la poche, le suivi et l'implosion des poches fractionnées.

Une exploitation plus fine des calculs par comparaison avec l'expérience va permettre de délimiter le domaine de validité du modèle d'Euler et d'examiner la nécessité d'améliorer le critère sur le transfert de masse.

Une autre extension possible du modèle consisterait à prendre en compte le couplage avec l'écoulement gazeux (considéré comme un fluide incompressible), ce qui permettrait d'améliorer la modélisation de la dynamique propre des poches de gaz notamment lorsque celles-ci sont fractionnées et soumises à des efforts tourbillonnaires importants.

\section{REMERCIEMENT}

Cette étude a été réalisée avec le soutien de la DRET.
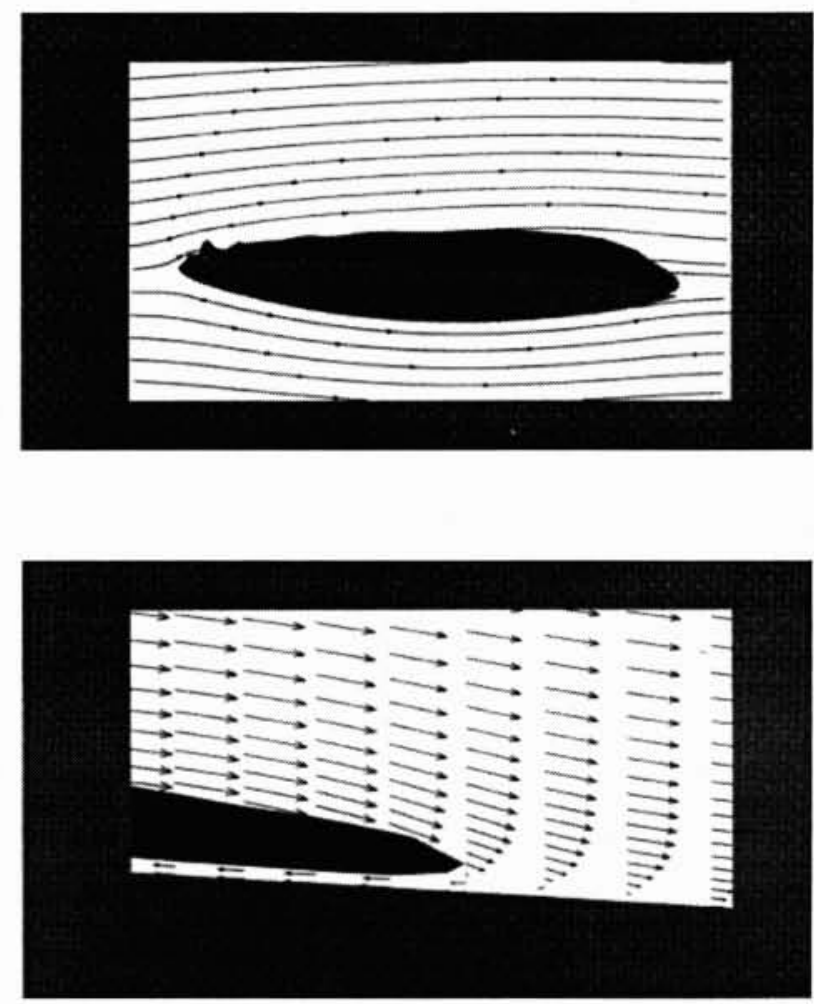

\section{BIBLIOGRAPHIE}

[1] Kubota A., Kato H. et Yamaguch H. - « Finite Difference Analysis of Unsteady Cavitation on a Two-Dimensionnal Hydrofoil ", 5th International Conference on Numerical Ship Hydrodynamics, Hiroshima, Japan, 1989.

[2] Ventikos Y., Tzabiras G. - « A numerical study of the steady and unsteady cavitation phenomenon around hydrofoils ", International Symposium on Cavitation, CAV'95, 2-5 may Deauville France, 1995.

[3] Delannoy Y. - * Modélisation d'écoulements instationnaires et cavitants ", thèse de l'Institut National Polytechnique de Grenoble, 1989.

[4] HIRT C.W. et. Nichols B.D. - « Volume Of Fluid (VOF) Method for the Dynamics of Free Boundaries ", Journal of Comp. Physics, Volumes 39, pages 201-255, 1981.

[5] De Jouette C., Viviand H., Wornom S. et Le Gouez J.M. - «Pseudo compressibility method for incompressible flow calculation ", 4th International Symposium on Comp. Fluid Dyn., Univ. of California at Davis, sept. 9-12, 1991.

[6] MARCER R. - «Validation sur résultats expérimentaux d'un modèle de simulation d'écoulements à surface libre non linéaire ", $12^{e}$ Congrès Français de la Mécanique, Strasbourg, 1995.

[7] JAMESON A., SCHMidT A., TURKel E. - Numerical Solutions for the Euler Equations by finite volumes methods using RungeKutta Time-Marching Schemes ", AIAA-81-1259, AIAA 14th Fluid and Plasma Dyn., Conf. Palo Alto, California, June 1981.

[8] De Jouette C. - « Développement d'une méthode de pseudo-compressibilité pour le calcul des écoulements de fluide incompressible. Applications aux écoulements instationnaires en présence d'une surface libre ", Thèse de l'Université de Nice. Sophia Antipolis, 1994.

[9] LE QUANG. - « Etude physique du comportement des poches de cavitation partielle », Thèse de doctorat de l'Institut National Polytechnique de Grenoble, 1989. 